\title{
BUFFERING TECHNIQUE FOR OBTAINING INCREASED PHYSIOLOGICAL RESPONSE FROM ALKALOIDAL EYE-DROPS*
}

\author{
BY \\ J. BOBERG-ANS, K. V. GROVE-RASMUSSEN, \\ and E. R. HAMMARLUND
}

From the Eye Department and Dispensary of the Finsen Institute and Radium Centre, and the Pharmacy Department, Royal Danish School of Pharmacy, Copenhagen

IT is widely accepted that the physiological activity of alkaloidal eye preparations results from the penetration through the cornea of the free base portion rather than of the ionic form of the alkaloid (Cogan and Kinsey, 1942; Cogan and Hirsch, 1944; Swan and White, 1942). Therefore, the smaller the $\mathrm{pKb}$ value of the alkaloid, the fewer free base particles will be available for drug action at the normal eye $\mathrm{pH} 7 \cdot 4$. This necessitates the use of larger doses to obtain the desired physiological response for many alkaloids. Moreover, the instability of alkaline aqueous solutions of the alkaloidal drugs limits their usefulness.

Since alkaloidal eye-drops are extensively used in eye examinations and treatment and are so potent that the lowest possible concentrations are desirable, efficient methods of using them have been much discussed.

Blok (1945) prepared eye-drops in alkaline buffer, $\mathrm{pH} 8-9$, in order to use smaller quantities of alkaloids, which were scarce during the German occupation of Holland. He found that a 0.5 per cent. alkaline pilocarpine solution produced a response similar to that from the usual 2 per cent. pilocarpine solution. However, the alkaline solution of the alkaloid was much less stable than the acidic solution.

Floyd, Kronfeld, and McDonald (1953) measured the miotic response of subjects who had 1 per cent. pilocarpine hydrochloride, $\mathrm{pH} 4 \cdot 0-4 \cdot 2$, instilled into one eye and the same solution buffered at $\mathrm{pH} \mathrm{6.6-7.4} \mathrm{into} \mathrm{the} \mathrm{other.}$ They found that the rate of absorption of the drug at each $\mathrm{pH}$ was approximately the same, although the more alkaline solution produced a slightly increased response.

Riegelman and Vaughan (1958), in an extensive discussion of the subject of ophthalmic solutions, compared the miotic response to 1 per cent. pilocarpine solutions buffered at $\mathrm{pH} 4 \cdot 2$ with that to those buffered at $\mathrm{pH} 6 \cdot 6$. They likewise found that pilocarpine in the less acid solution had slightly more miotic effect but that the difference was statistically insignificant.

One might. assume that these results were due to the fact that there was not enough additional alkaloidal free base available for increased penetration of the cornea at the highest $\mathrm{pH}$ used in the experiments.

\footnotetext{
* Received for publication January 29, 1959.
} 
In the present investigations it was found that, if an alkaloidal salt is dissolved in sterile water and made isotonic with sodium chloride and is then temporarily or even momentarily buffered in the eye at the sufficiently alkaline $\mathrm{pH}$ of $9 \cdot 2$, considerably more free base is available and will produce a markedly increased physiological action. This is true for many alkaloidal drugs. It is proposed to describe a technique whereby one can instill into the eye a considerably smaller concentration of various stable, acidic, aqueous solutions of alkaloidal salts and still obtain the desired physiological effect as rapidly and fully as that obtained with the higher concentrations of acidic buffered solutions that are frequently used. This is accomplished by first "buffering" the eye by instilling one or two drops of a sterile isotonic sodium borate solution, $\mathrm{pH} 9 \cdot 2$, into the eye, and by following this with one drop of the sterile aqueous solution of the desired alkaloidal salt made isotonic with sodium chloride. The buffer solution has the following composition:

$$
\begin{array}{ll}
\text { Sodium borate (analytical) } & \mathbf{0 . 7 8} \mathbf{~ g} . \\
\text { Sterile distilled water } & \text { q.s. } 30 \mathrm{ml} \text {. }
\end{array}
$$

Sodium borate solution was considered to be a suitable buffer because it has been used frequently in the eyes and has a sufficiently high buffer capacity. For example, when ten volumes of 0.5 per cent. atropine sulphate aqueous solution are added to one volume of the borax buffer solution, the $\mathrm{pH}$ of the resulting mixture is $9 \cdot 1$.

The pupil response is the criterion of the pharmacological effect of the alkaloidal drug. The concentrations used after the instillation of the buffer solution are from one-half to one-twentieth of those normally employed for average physiological activity without use of the buffer.

\title{
Method
}

The following drugs were used in aqueous isotonic solution at the given concentrations:

\author{
Homatropine hydrobromide $0 \cdot 1$ per cent. \\ Phenylephrine hydrochloride 1 per cent. \\ Ephedrine hydrochloride 1 per cent. \\ Atropine sulphate $\mathbf{0} \cdot 1$ per cent. \\ Pilocarpine hydrochloride 0.4 per cent. \\ Tetracaine hydrochloride $0 \cdot 1$ per cent.
}

The first three drugs were individually tested on human subjects in the laboratory before being submitted to clinical evaluation at the Eye Clinic of the Finsen Institute and Radium Centre. The other three were evaluated only by clinical investigation.

The preliminary tests, in which each drug was used on at least six persons, were carried out as follows:

One drop of a sterile isotonic solution of sodium borate was instilled just above the cornea in one eye, the experimental eye, of each subject. Immediately one drop of the 
alkaloidal solution under study was placed in both eyes of the test subjects. The eye which received an identical drop of the alkaloidal solution but with no previous buffer application served as the control. The examinee was advised to blink normally after all administrations. The horizontal diameter in $\mathrm{mm}$. of the pupil of each eye was measured before the drug administration and every $15 \mathrm{~min}$. thereafter for $3 \mathrm{hrs}$. The measurements were made with a ruler to the nearest $0.5 \mathrm{~mm}$., the subject being placed in the same position each time in an evenly lighted room.

\section{Results}

For each drug the results were plotted as $\mathrm{mm}$. diameter for the right and left pupils by the time in minutes. The mean average mydriatic response obtained from the subjects tested is shown in Figs 1 to 3.

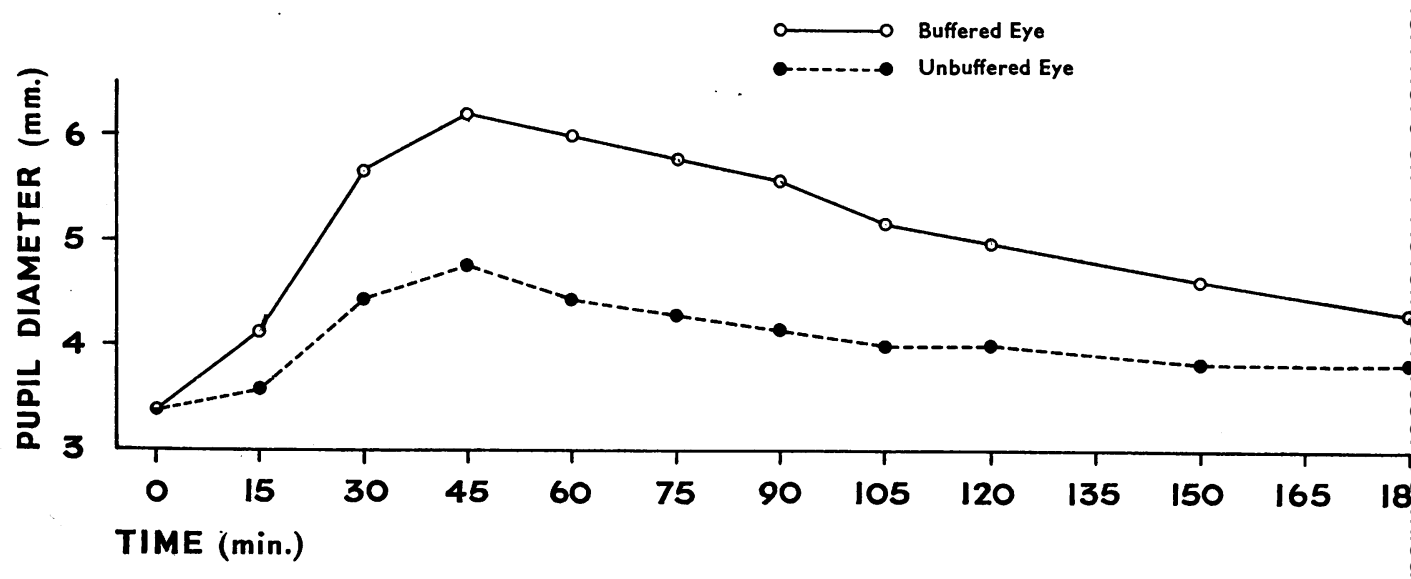

FIG. 1.-Mydriatic effect from 1 per cent. ephedrine $\mathrm{HCl}$ solution.

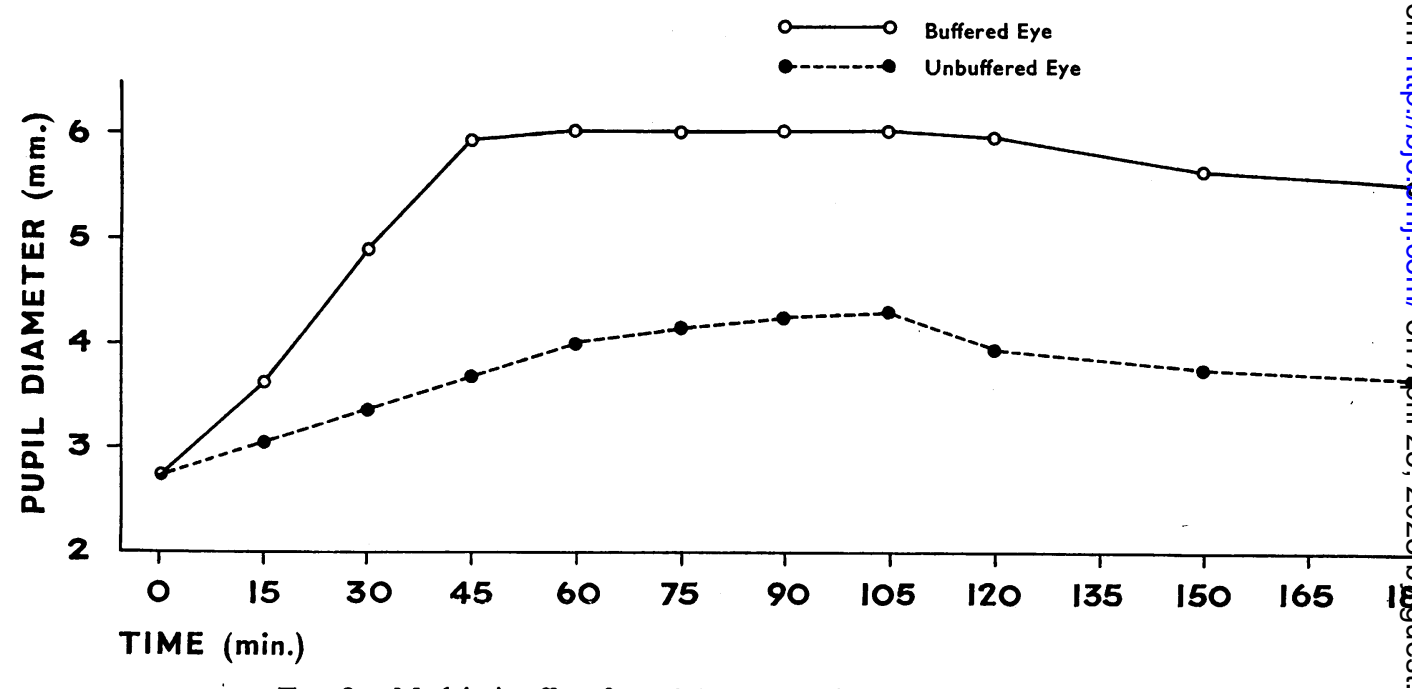

Fig. 2.-Mydriatic effect from $0 \cdot 1$ per cent. homatropine $\mathrm{HBr}$ solution. 


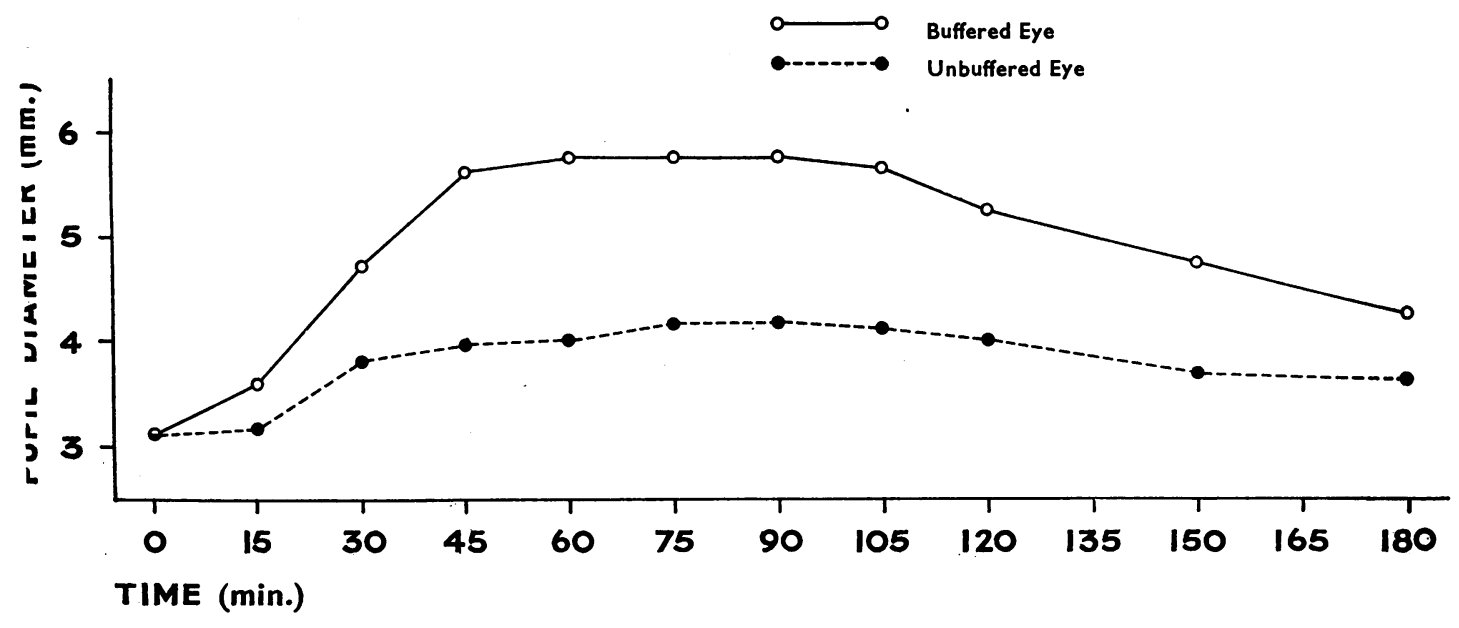

FIG. 3.-Mydriatic effect from 1 per cent. phenylephrine $\mathrm{HCl}$ solution.

In another laboratory experiment on six individuals, the mydriatic response to a $0 \cdot 1$ per cent. homatropine hydrobromide solution after the instillation of a sodium borate solution was compared with that to a 1 per cent. homatropine hydrobromide solution in the other eye with no buffer. The test was carried out as before, and it was found that the weaker solution plus the buffer gave the same amount of pupil dilation as the stronger solution with no buffer (Fig. 4). However, this should not necessarily be interpreted as meaning that there was a ten-fold increase in drug action, because near maximum pupil dilation was reached in both cases.

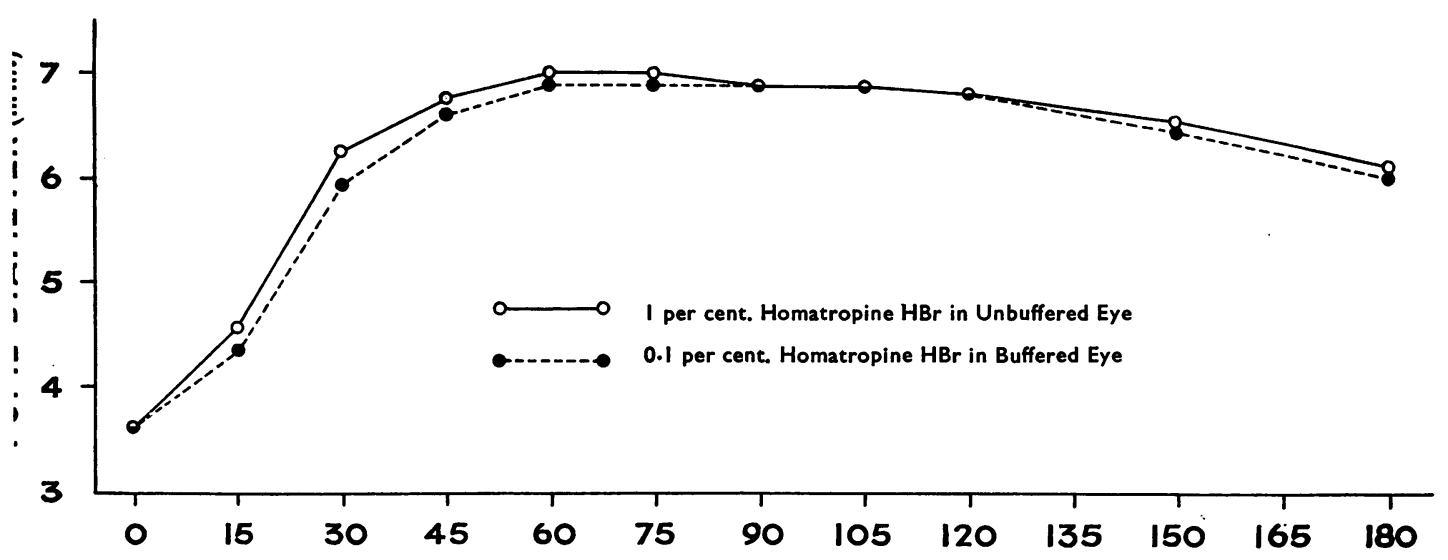

TIME (min.)

FIG. 4.-Mydriatic effect from 0.1 per cent. and 1 per cent. homatropine $\mathrm{HBr}$ solutions.

Mueller and Deardorff (1956) reported that the addition of 1 per cent. methyl cellulose 4000 to a $0 \cdot 25$ per cent. homatropine hydrobromide solution produced an increased mydriatic response in the eyes. Further experiments were therefore made on two subjects to find out whether the addition of 1 per cent. methyl cellulose 
4000 to the sodium borate buffer solution would increase further the mydriatic effect of 0.1 per cent. homatropine hydrobromide solution when used with the buffer pre-treatment. Tests made in the same manner as before revealed a small increase in mydriatic response, although it was too small to be of significance.

\section{Clinical Investigation}

$0 \cdot 1$ per cent. homatropine hydrobromide, 2 per cent. phenylephrine hydrochloride, $0 \cdot 1$ per cent. atropine sulphate, and $0 \cdot 4$ per cent. pilocarpine hydrochloride were clinically tested by dropping the solutions into the eyes of patients at the Eye Clinic as follows:

One eye was pre-treated with two drops of borax buffer solution, and one drop of the solution tested was instilled into each eye. In every case the response was quicker in the pre-treated eye; the reaction was more pronounced, and the maximum effect lasted longer than in the unbuffered eye. This was true in patients of all ages.

Atropine, homatropine, and phenylephrine solutions used at the full usual strength in combination with sodium borate had no untoward effect in adults (it was not tried in children below the age of 10). Although the effect was more rapid, the maximum reached was not greater than in the eyes treated without sodium borate.

The speed of penetration through the cornea into the anterior chamber and the effect on the structures here seems to be considerably enhanced by the alkaline-buffered solution of sodium borate. The influence on the superficial sensitive nerves in the cornea seems to be equal for both acidic and alkaline solutions. After the cornea had been anaesthetized with $0 \cdot 1$ per cent. solution of tetracaine hydrochloride, the corneal sensitivity was followed with the Boberg-Ans $(1955,1956)$ sensibilometer. The anaesthetic took effect in the same time in eyes pre-treated with the borate buffer as in eyes not pre-treated. The depth of anaesthesia was also equal, but the duration was prolonged for some minutes in the buffered eyes.

This buffering technique is now frequently used whenever eye treatment is required before surgery. No cases of atropine or other alkaloidal toxic effects have been observed at the Eye Clinic.

In conclusion, it should be stated that a weak solution of one-half to one-twentieth of the concentration usually used is just as effective as the stronger solutions when the conjunctival sac has been buffered with sodium borate solution.

Hind and Goyan (1947) reported that the free base of the alkaloid caused the irritation sometimes associated with eye-drops. However, Trolle-Lassen (1958) stated that the

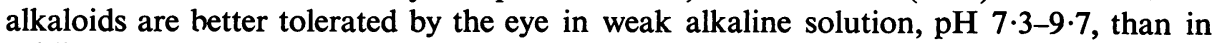
acidic solution provided they are approximately isotonic with tears. Martin and Mims (1950) stated that whether or not a particular $\mathrm{pH}$ value of a solution will prove to be a source of irritation must be determined for any given combination of ions and substances in solution. In the present investigation there was no evidence of pain after the instillation of aqueous alkaloidal salt solutions into the "buffered" eyes, except that the 1 per cent. ephedrine hydrochloride solution produced a mild stinging sensation for a very few' seconds in the eyes of some subjects.

Cogan and Hirsch (1944) called attention to the undesirable systemic side-effects which sometimes follow the administration of potent ophthalmic alkaloidal solutions. Therefore, since much weaker solutions are required, the use of the buffering technique described herein may frequently be advantageous.

Summary

A technique is described by which the normal physiological response may be obtained from a much weaker concentration of alkaloidal eye-drops than 
is usually required. An isotonic, sterile 2.6 per cent. sodium borate solution is instilled before the eye-drops, which can then be used without discomfort to the patient. The addition of 1 per cent. methyl cellulose 4000 to the borax buffer solution had no significant effect.

One of us (E.R.H.) wishes to thank the School of Pharmacy, State College of Washington, Pullman, U.S.A., from where he is on sabbatical leave of absence, and also the Gustavus A. Pfeiffer Memorial Research Fellowship for the 1958-59 award.

\section{REFERENCES}

BLoK, C. J. (1945). Pharm. J., 155 (4 ser., 101), 282.

BOBERG-ANS, J. (1955). Brit. J. Ophthal., 39, 705. (1956). Acta ophthal. (Kbh.), 34,149.

Cogan, D. G., and Hirsch, E. O. (1944). Arch. Ophthal. (Chicago), 32, 276.

and KINSEY, V. E. (1942). Ibid., 27, 466, 696.

Floyd, G., Kronfeld, P. C., and McDonald, J. E. (1953). J. Amer. pharm. Ass., Sci. Ed., 42, 333.

HIND, H. W., and Goyan, F. M. (1947). Ibid., 36, 33.

Martin, F. N., and Mims, J. L. (1950). Arch. Ophthal. (Chicago), 44, 561.

Mueller, W. H., and DeARDORFF, D. L. (1956). J. Amer. pharm. Ass., Sci. Ed., 45, 334.

Riegelman, S., and Vaughan, D. G. (1958). Ibid., Pract. Pharm. Ed., 19, 474.

SwAN, K. C., and White, N. G. (1942). Amer. J. Ophthal., 25, 1043.

Trolle-Lassen, C. (1958). Pharm. Weekbl., 93, 148. 